\title{
Classically and alternatively activated bone marrow derived macrophages differ in cytoskeletal functions and migration towards specific CNS cell types
}

Elly JF Vereyken ${ }^{1}$, Priscilla DAM Heijnen ${ }^{1}$, Wia Baron², Elga HE de Vries ${ }^{1}$, Christine D Dijkstra $^{1 *}$ and Charlotte E Teunissen ${ }^{3}$

\begin{abstract}
Background: Macrophages play an important role in neuroinflammatory diseases such as multiple sclerosis (MS) and spinal cord injury (SCl), being involved in both damage and repair. The divergent effects of macrophages might be explained by their different activation status: classically activated (CA/M1), pro-inflammatory, macrophages and alternatively activated (AA/M2), growth promoting, macrophages. Little is known about the effect of macrophages with these phenotypes in the central nervous system (CNS) and how they influence pathogenesis. The aim of this study was therefore to determine the characteristics of these phenotypically different macrophages in the context of the CNS in an in vitro setting.

Results: Here we show that bone marrow derived CA and AA macrophages have a distinct migratory capacity towards medium conditioned by various cell types of the CNS. AA macrophages were preferentially attracted by the low weight $(<10 \mathrm{kD}$ ) fraction of neuronal conditioned medium, while CA macrophages were attracted in higher numbers by astrocyte- and oligodendrocyte conditioned medium. Intrinsic motility was twice as high in AA macrophages compared to CA macrophages. The adhesion to extracellular matrix molecules (ECM) was significantly enhanced in CA macrophages compared to control and AA macrophages. The actin cytoskeleton was differentially organized between CA and AA macrophages, possibly due to greater activity of the GTPases RhoA and Rac in CA macrophages. Phagocytosis of myelin and neuronal fragments was increased in CA macrophages compared to AA macrophages. The increase in myelin phagocytosis was associated with higher expression of CR3/ MAC-1 in CA macrophages.

Conclusion: In conclusion, since AA macrophages are more motile and are attracted by NCM, they are prone to migrate towards neurons in the CNS. CA macrophages have a lower motility and a stronger adhesion to ECM. In neuroinflammatory diseases the restricted migration and motility of CA macrophages might limit lesion size due to bystander damage.
\end{abstract}

Keywords: migration, classically activated macrophages, alternatively activated macrophages, central nervous system, neurons

\footnotetext{
* Correspondence: cd.dijkstra@vumc.nl

'Department of Molecular Cell Biology and Immunology, VU University

Medical Center, Amsterdam, the Netherlands

Full list of author information is available at the end of the article
} 


\section{Background}

Macrophages are phagocytic cells that play an essential role in both innate and acquired immunity. Macrophages are not a homogeneous cell population, since they are highly plastic cells that are able to respond to a variety of environmental cues by changing their phenotype and physiology $[1,2]$. The two phenotypes that are considered to be the most extreme are classically activated (CA/M1) pro-inflammatory macrophages and alternatively activated (AA/M2) or growth promoting macrophages. In tissues, the micro-environment of the macrophages is thought to determine the phenotype [2]. In vitro, cytokines and other stimuli induce these activation phenotypes. CA macrophages are induced by interferon-gamma (IFN- $\gamma$ ) and lipopolysaccharide (LPS). The induction of the AA phenotype is not straightforward, due to the fact that a range of stimuli, such as IL-4/IL13, IL-10, immunocomplexes and glucocorticoids, are reported to induce alternative activation in macrophages [2]. Consequently, a wide variety of phenotypical and functional characteristics have been attributed to alternatively activated macrophages. The most common and widely studied way to generate AA macrophages is by exposure to interleukin (IL)-4/IL-13 [1,3]. Notably, IL-4 stimulated AA and CA macrophages have distinct functions in tissue repair and inflammation. The CA macrophages produce nitric oxide $(\mathrm{NO})$ and reactive oxygen species (ROS) making them cytotoxic $[1,4]$. Furthermore, they secrete high amounts of pro-inflammatory cytokines, for example IL-12, that promote inflammation. Conversely, AA macrophages produce anti-inflammatory cytokines, such as IL-10 $[1,2]$, thereby reducing inflammation. AA macrophages have a higher angiogenic potential compared to CA macrophages [5]. They produce growth factors, such as platelet derived growth factor (PDGF), vascular endothelial growth factor (VEGF) and fibroblast growth factor (FGF) [5-7]. In mice, AA macrophages express high levels of arginase. Due to activation of arginase, arginine is converted to ornithine, a precursor for collagen, which contributes to the production of extracellular matrix molecules (ECM) [8-11] and promotes cell growth $[10,12]$. Together these features determine the wound healing and growth promoting phenotype of AA macrophages [1,2].

Macrophages can play crucial roles during neuroinflammatory diseases, such as spinal cord injury (SCI) and multiple sclerosis (MS). MS is a chronic inflammatory disease of the central nervous system (CNS). Major neuropathological hallmarks of MS are inflammatory demyelinating lesions associated with perivascular infiltrates containing macrophages and lymphocytes $[13,14]$. It is widely accepted that macrophages play an important role during MS pathogenesis and both detrimental and beneficial effects of macrophages have been observed during MS and experimental autoimmune encephalomyelitis (EAE), an animal model for MS. Activated macrophages secrete many products that could contribute to axonal and oligodendrocyte damage seen in MS lesions, such as matrix metalloproteinases (MMPs) [15], NO and glutamate, [16-22]. The elimination of infiltrating macrophages by clodronate liposomes suppressed clinical signs of EAE [23,24]. A correlation was found between location and amount of axonal damage and loss and cellular infiltrates containing macrophages [25-27]. These studies suggest that macrophages play a detrimental role, but beneficial effects have been reported for macrophages as well. Phagocytosis of myelin debris is important for axonal repair/ regrowth and remyelination, since myelin components are known to be growth inhibiting [28,29]. Moreover, activated macrophages/microglia are found to be sources of neurotrophins and growth factors, such as brain derived neurotrophic factor (BDNF) in MS lesions $[30,31]$ and elimination of infiltrating macrophages reduced remyelination in demyelinating models [32].

These divergent effects of macrophages have also been observed during SCI. As with MS, depletion of infiltrating macrophages was beneficial, indicating that macrophages are detrimental. Elimination of infiltrating macrophages was observed to increase axonal repair and functional outcome [33-35]. However, macrophages can also create a growth-permissive environment in which axonal regeneration can take place during SCI [36-38]. Kigerl et al. observed that CA macrophages predominate in the SCI lesion, while the AA macrophage response is only transient in lesions, possibly explaining limited repair [39].

The divergent effects of macrophages in neuroinflammatory diseases might be due to the different activation states that macrophages can acquire. Little research has been done on AA and CA macrophages and their functions in the CNS environment. Kigerl et al. found that conditioned medium from CA macrophages was toxic to neurons, while conditioned medium from AA macrophages was not and could even induce axonal outgrowth across a gradient of inhibitory substrate [39]. Microglia activated by IL- 4 are able to support oligodendrogenesis and some neurogenesis from adult neuronal progenitor cells, indicating a role in CNS repair for these microglia [40]. Furthermore, in microglia IL-4 induces the expression of genes typical for alternative activation and IL-4 deficiency in the CNS led to exacerbation of EAE [41].

The aim of the current study was to determine the functional characteristics of CA and AA macrophages, with respect to migration, motility, adhesion and phagocytosis, in the context of the CNS. We demonstrate for the first time that $\mathrm{CA}$ and AA macrophages behave differently under influence of the conditioned media of 
CNS cells. Furthermore, motility, adhesion and phagocytosis, cytoskeletal functions, varied between CA and AA macrophages. This was due to variation in cytoskeletal organization, activity of RhoA and Rac, and ROS production.

\section{Materials and methods \\ Animals}

For neuronal and astrocyte cultures, timed pregnant C57BL/6 mice were obtained from Charles River (Maastricht, The Netherlands). For isolation of bone marrow, adult C57BL/6 mice were used from Charles River.

All experiments were performed according to the guidelines of the local University Committee on Animal Welfare, which follow the European Communities Council Directive (86/609/EEC).

\section{Macrophages}

Bone marrow derived macrophages were generated as described previously [42]. Bone marrow was flushed from femurs and tibias of C57/BL6 mice and cultured for 1 week in complete macrophage medium (Dulbecco modified Eagle's minimal essential medium (DMEM) (Invitrogen, Breda, the Netherlands) supplemented with $10 \%$ fetal calf serum (FCS) (Invitrogen), 15\% conditioned medium from macrophage-colony stimulating factorsecreting L929 fibroblasts and 2\% penicillin/streptomycin-glutamine (Lonza, Breda, the Netherlands) at $37^{\circ} \mathrm{C}$. After 7-10 days in culture adherent cells were approximately $95 \%$ pure macrophages and cells were used for experiments.

The CA phenotype was induced by exposing macrophages for $48 \mathrm{hr}$ to $5 \times 10^{3} \mathrm{U} / \mathrm{ml}$ IFN- $\gamma$ (U-Cytech, the Netherlands) and $10 \mathrm{ng} / \mathrm{ml}$ Escherichia coli LPS (026:B6; Sigma-Aldrich, Zwijndrecht, the Netherlands) in the culture medium. AA macrophages were prepared by exposure to $10 \mathrm{ng} / \mathrm{ml} \mathrm{IL-4} \mathrm{(Invitrogen)} \mathrm{in} \mathrm{the} \mathrm{culture}$ medium $[3,43]$. Control macrophages were cultured for the same period in medium alone.

Macrophages were harvested by 15 min incubation at $37^{\circ} \mathrm{C}$ with lidocaine ( $4 \mathrm{mg} / \mathrm{ml}$ in PBS; Sigma-Aldrich). Macrophages were washed and centrifuged for $5 \mathrm{~min}$ at $170 \mathrm{~g}$.

The phenotype of the differently activated macrophages was determined using a Griess assay and FACS analysis. Using the Griess assay, nitrite, the stable end product of NO, was measured. $100 \mu \mathrm{l}$ supernatant of the differently activated macrophages was added to 100 $\mu \mathrm{l}$ Griess reagent $(0.1 \%$ anphthylene diamine dihydrochloride, $1 \%$ sulfanilamide and $2.5 \% \mathrm{H} 3 \mathrm{PO} 4)$. The OD540 was measured on a Benchmark microplate reader (Bio-Rad laboratories, Veenendaal, the Netherlands) and the concentration of nitrite present in the supernatant was determined by linear regression from a standard curve using known concentrations of sodium nitrite.

\section{FACS analysis}

To determine the expression of several markers on differently activated macrophages a FACS analysis was performed. In brief, $1 * 10^{5}$ macrophages were washed and incubated with the first antibody (see Table 1) for one h. Subsequently, macrophages were washed and exposed to the secondary fluorescently labeled antibody (see Table 1) for one h. The extent of marker expression was analysed using flow cytometry (FACSCalibur, Becton Dickinson, Erembodegem, Belgium) combined with Cellquest Pro software (Becton Dickinson). Omission of the primary antibody was included as negative control. The mean fluorescent intensity (MFI) of the macrophages was measured and data of three separate experiments were averaged. To obtain a better insight into the relative upregulation of the markers on the differently activated macrophages the results were expressed relative to control macrophages.

\section{Conditioned media}

Neuron conditioned medium (NCM) was derived from neurons of C57/BL6 primary mouse CNS. Embryonic day 19 mouse pups were sacrificed and the brain was isolated. For neuronal cultures, the cortex was isolated and incubated with trypsin containing $0.1 \mathrm{mg} / \mathrm{ml}$ DNAse for $15 \mathrm{~min}$ at $37^{\circ} \mathrm{C}$. The cell suspension was extensively washed and the neurons were triturated to create a single cell suspension. Neurons were cultured in complete neurobasal medium, consisting of incomplete neurobasal medium with 1\% glutamax, 2\% B27 and $0.01 \%$ gentamycin (all obtained from Invitrogen), at a concentration of $1 \times 10^{5}$ cells $/ \mathrm{ml}$. Beta-tubulin (Covance, Uden, the Netherlands) staining was performed and cultures were found to be approximately $90 \%$ pure (data not shown, rest are predominantly astrocytes and some microglia). After 2 days in culture NCM was harvested.

For astrocyte conditioned medium (ACM), the forebrain cortex of mouse pups was isolated and single cell suspension was generated. Cells were cultured for 1 week in complete medium which consisted of DMEM with high glucose, supplemented with $1 \%$ glutamax, $10 \%$ FCS and $0.01 \%$ gentamycin (all obtained from Invitrogen). After 1 week, new medium was added to the culture. Cultures were characterized using glial fibrillary acidic protein (GFAP, Sigma-Aldrich) staining and were found to be approximately 95\% pure (rest are neurons and microglia, data not shown). After $48 \mathrm{hr}$ of culturing, ACM was harvested.

Oligodendrocyte conditioned medium (OCM) was harvested during oligodendrocyte development. Primary 
Table 1 Antibodies and dilutions used in FACS analysis of macrophages

\begin{tabular}{lllll}
\hline Primary antibody & Subtype & Dilution & Secondary antibody & Dilution \\
\hline F4/80 (Serotec, Oxford, UK) & $\operatorname{lgG}$ & $1: 500$ & $\begin{array}{l}\text { Anti-rat Alexa 488 } \\
\text { (Invitrogen) }\end{array}$ & $1: 400$ \\
\hline MAC-1 (Serotec, Oxford, UK) & $\operatorname{lgG}$ & $1: 500$ & $\begin{array}{l}\text { Anti-rat Alexa-488 } \\
\text { (Invitrogen) }\end{array}$ \\
\hline MR biotinylated (Biolegend, Uithoorn, the Netherlands) & $\lg$ & $1: 100$ & $\begin{array}{l}\text { Streptavidin-Alexa 488 } \\
\text { (Invitrogen) }\end{array}$ & $1: 400$ \\
\hline LFA-1 produced in house & $\operatorname{lgG}$ & $1: 250$ & $\begin{array}{l}\text { Anti-rat Alexa 488 } \\
\text { (Invitrogen) }\end{array}$ & $1: 400$ \\
\hline CD29 (BD Pharmingen, Breda, the Netherlands) & $\operatorname{lgG}$ & $1: 100$ & $\begin{array}{l}\text { Anti-rat Alexa 488 } \\
\text { (Invitrogen) }\end{array}$ & $1: 400$ \\
\hline CD11a-FITC (eBioscience, Malden, the Netherlands) & $\operatorname{lgG}$ & $1: 100$ & & \\
\hline
\end{tabular}

rat oligodendrocytes were cultured as described previously [44]. Briefly, oligodendrocyte precursors were cultured on poly-l-lysine coated cell culture plates for 2 days in a defined SATO medium [45] containing platelet derived growth factor-AA (PDGF-AA) and fibroblast growth factor-2 (FGF-2) in order to synchronize precursors to the bipolar oligodendrocyte-type II astrocyte $(\mathrm{O} 2 \mathrm{~A})$ stage. Differentiation was induced by replacing the growth factors with $0.5 \%$ FCS in SATO medium. Medium was harvested from: (i) cells in the O2A stadium (designated as O2A medium); (ii) cells that had differentiated from O2A to the galactocerebroside (GC) stage (3 days differentiation, GC medium); (iii) cells that differentiated from $\mathrm{GC}$ to myelin basic protein (MBP) positive stage (7 days differentiation, MBP medium); finally from cells that had developed from MBP to MBP + stage (10 days differentiation, designated MBP + medium).

\section{Fractioning of the conditioned media}

In order to get insight into the range of molecular weight of the factors responsible for attraction of macrophages, conditioned media were fractioned based on molecular weight. Aliquots of the conditioned media were filtered using 10, 50 and $100 \mathrm{~K}$ Amicon Ultra centrifugal filter units as described by the manufacturer (Millipore, Amsterdam, the Netherlands). The aliquots were first filtered using the $10 \mathrm{kD}$ filter, subsequently 50 and $100 \mathrm{kD}$, creating three fractions: one containing low molecular weight $(<10 \mathrm{kD})$, one with intermediate molecular weight (between 10 and $50 \mathrm{kD}$ ) and a fraction from 50 to $100 \mathrm{kD}$. The fractions were reconstituted in half of the original volume.

\section{Migration and motility}

The migratory capacity of CA and AA macrophages was studied using a 48-wells micro chemotaxis chamber (Neuro Probe, Gaithersburg, USA) as described previously [46] with some modifications. In the bottom well, $25 \mu \mathrm{l}$ of the conditioned media, control medium (neurobasal for NCM, DMEM for ACM and SATO medium for OCM), monocyte chemotactic peptide-1 (MCP-1) (20 ng/ml; Peprotech inc, London, UK) or formyl methionineleucyl-phenylalanine (fMLP) (10 nM; Sigma-Aldrich) was added. A filter with a pore size of $10 \mu \mathrm{m}$ was used. In the upper chamber $2 * 10^{4}$ differently activated macrophages were added. The macrophages were left to migrate for $6 \mathrm{~h}$. The side of the filter in direct contact with the upper chamber was washed and scraped clean of cells and the filter was subsequently stained using Coommassie Blue. The number of migrated cells was counted per $0.1 \mathrm{~mm}^{2}$ using a scored eyepiece. From this the total number of migrated cells was calculated.

To determine motility, $5 * 10^{4} \mathrm{CA}$ and AA macrophages were seeded in culture plastic 96 wells plate (Greiner Bio One, Alphen a/d Rijn, the Netherlands) and cultured for $1 \mathrm{~h}$. The 96-wells plate was placed in a time-lapse video microscope. Macrophages in each well were followed for $15 \mathrm{~min}$ and images were taken every 20 seconds at a 40 times magnification. The software program Track-It ${ }^{\mathbb{B}}$ was used to calculate speed and distance moved as a measure for motility. All the cells present in a microscopic field were tracked manually.

\section{Adhesion}

Macrophage adhesion to plastic and several extracellular matrix molecules (ECM) was determined as described previously [47]. CA and AA macrophages were harvested and labeled with $1 \mu \mathrm{M}$ BCECF-AM (Invitrogen) for 15 min at $37^{\circ} \mathrm{C}$. After labeling, cells were washed and $1 \times$ $10^{5}$ macrophages in $100 \mu \mathrm{l}$ were seeded in the 96 wells plates. Macrophages were left to adhere for $2 \mathrm{~h}$ at $37^{\circ} \mathrm{C}$ and $5 \% \mathrm{CO}_{2}$. After incubation, non-adherent cells were removed by washing 3 times in PBS and the remaining adherent cells were lysed with $0.1 \mathrm{~N} \mathrm{NaOH}$. Fluorescence was measured in a Fluostar24 (BMG labtechnologies, Offenburg, Germany). A standard curve with cell concentrations ranging from $5 \times 10^{3}$ to $1 \times 10^{6}$ cells $/ \mathrm{ml}$ was made to determine the percentage of adhering cells. 
To determine the adherence of differently activated macrophages to different ECM molecules, wells of a 96-well culture plate were coated with either collagen (type I from calf skin, Sigma-Aldrich), for $1 \mathrm{~h}$ at room temperature, or fibronectin (derived from human plasma; Roche, Almere, the Netherlands), for $1 \mathrm{~h}$ at $37^{\circ} \mathrm{C}$.

We investigated the expression of adhesion receptors on differently activated macrophages using FACS analysis. We performed the FACS analysis as described above. The antibodies used are listed in Table 1.

\section{Actin cytoskeleton}

The actin cytoskeleton of the differently activated macrophages was visualized using rhodamine phalloidin (Invitrogen). Macrophages were cultured on glass coverslips and stimulated in order to generate the different phenotypes. After 2 days, macrophages were fixed by incubation for $30 \mathrm{~min}$ with paraformaldehyde (4\% in PBS). The macrophages were washed twice with PBS containing 0.1\% Tween-20 (Sigma-Aldrich) and exposed to rhodamine phalloidin (1:300, Sigma-Aldrich) in PBS containing $0.1 \%$ Tween- 20 for $1 \mathrm{~h}$. To visualize nuclei, the cells were counterstained with Hoechst (Invitrogen) and afterwards embedded in mounting medium.

\section{RhoA and Rac activity G-LISA}

RhoA and Rac activity in differently activated macrophages was determined using G-LISA activation assay kit (Cytoskeleton, Denver, USA) according to the manufacturer's description. In brief, macrophage cell lysate was added to a pre-coated 96-wells plate to which the active, GTP-bound form of the protein will bind. The bound protein was detected using a primary antibody to the protein and a secondary antibody linked to HRP. The signal was developed using OPD and absorbance was measured using a Benchmark microplate reader (Bio-Rad laboratories, Veenendaal, the Netherlands).

\section{Phagocytosis}

The extent of phagocytosis of fluorescently labeled myelin and neuronal fragments by macrophages was determined using fluorescence activated cell sorter (FACS) analysis. Neuronal fragments were made by roughly pipetting 2 day old neuronal cultures, prepared as described previously [48], and labeling them with the lipophilic fluorescent dye 1,1',di-octadecyl-3,3,3'3'-tetramethylindocarbocyanine perchlorate (DiI) (SigmaAldrich). We adapted a protocol described previously for myelin phagocytosis ${ }^{41}$.

$3^{*} 10^{5}$ Macrophages were washed twice and incubated with fluorescently labeled myelin $(10$ or $20 \mu \mathrm{g} / \mathrm{ml})$ for $1.5 \mathrm{~h}$ at $37^{\circ} \mathrm{C}$. Macrophages were washed three times and harvested using $4 \mathrm{mg} / \mathrm{ml}$ lidocain (Sigma-Aldrich) for $15 \mathrm{~min}$ at $37^{\circ} \mathrm{C}$. The macrophages were washed and analyzed using flow cytometry and Cellquest Pro software. Phagocytosis was displayed in percentages relative to control macrophages.

To determine the effect of CR3, small GTPases and ROS on phagocytosis, anti-CR3 antibody $(10 \mu \mathrm{g} / \mathrm{ml})$, quercetin (300 $\mu \mathrm{M}$, Kaden Biochemicals, Hamburg, Germany), luteolin $(300 \mu \mathrm{M}$, Kaden Biochemicals) and diphenylene iodonium (DPI) (Sigma-Aldrich) were added to macrophages during the phagocytosis.

To exclude that the phagocytosis measured on the FACS is only binding, confocal images were taken of macrophages after phagocytosis. Macrophages were cultured and stimulated for $48 \mathrm{~h}$ with either IFN- $\gamma$ and LPS or IL-4 to generate the different phenotypes on cover slip glasses. The macrophages were incubated with DiI labeled myelin or neuronal fragments for $1.5 \mathrm{~h}$ at $37^{\circ} \mathrm{C}$. Afterwards, the macrophages were stained using MAC-1 and the Alexa 488 labeled anti-rat secondary antibody. Images were taken on a Leica TCS SP2 confocal microscope.

\section{Statistical analysis}

The data are expressed as mean of 3 to 4 separate experiments performed in duplo $( \pm$ SEM). Statistics were performed in SPSS (15.0.0, Chicago, USA). The motility, migration, adhesion, G-LISA and phagocytosis experiments were analysed using one-way ANOVA with Bonferroni correction. A p-value of less than 0.05 was considered significant.

\section{Results}

\section{Phenotype}

The phenotype of the differently activated macrophages was determined by investigating both marker expression and NO production. The expression of Mac-1, F4/80 and MR was determined using FACS analysis. MR is a longstanding marker for the AA phenotype [3]. Macrophages stimulated in vitro with IFN- $\gamma$ and LPS have been found to express high levels of Mac-1 [49]. In accordance with literature, we observed a significant upregulation of the immunoreactivity for Mac-1 and F4/ 80 on CA macrophages, while the immunoreactivity for MR was increased on AA macrophages (Figure 1A).

CA macrophages are known to produce NO and ROS. Therefore, the production of NO was taken as a marker for the CA phenotype. Significant amounts of NO were produced by $\mathrm{CA}$ macrophages, while no detectable amounts of NO were produced by AA and control macrophages (Figure 1B).

\section{CA and AA macrophages migrate differently towards conditioned media from CNS cells}

To investigate whether $\mathrm{CA}$ and $\mathrm{AA}$ macrophages migrate towards different CNS cell types, a 48-wells 


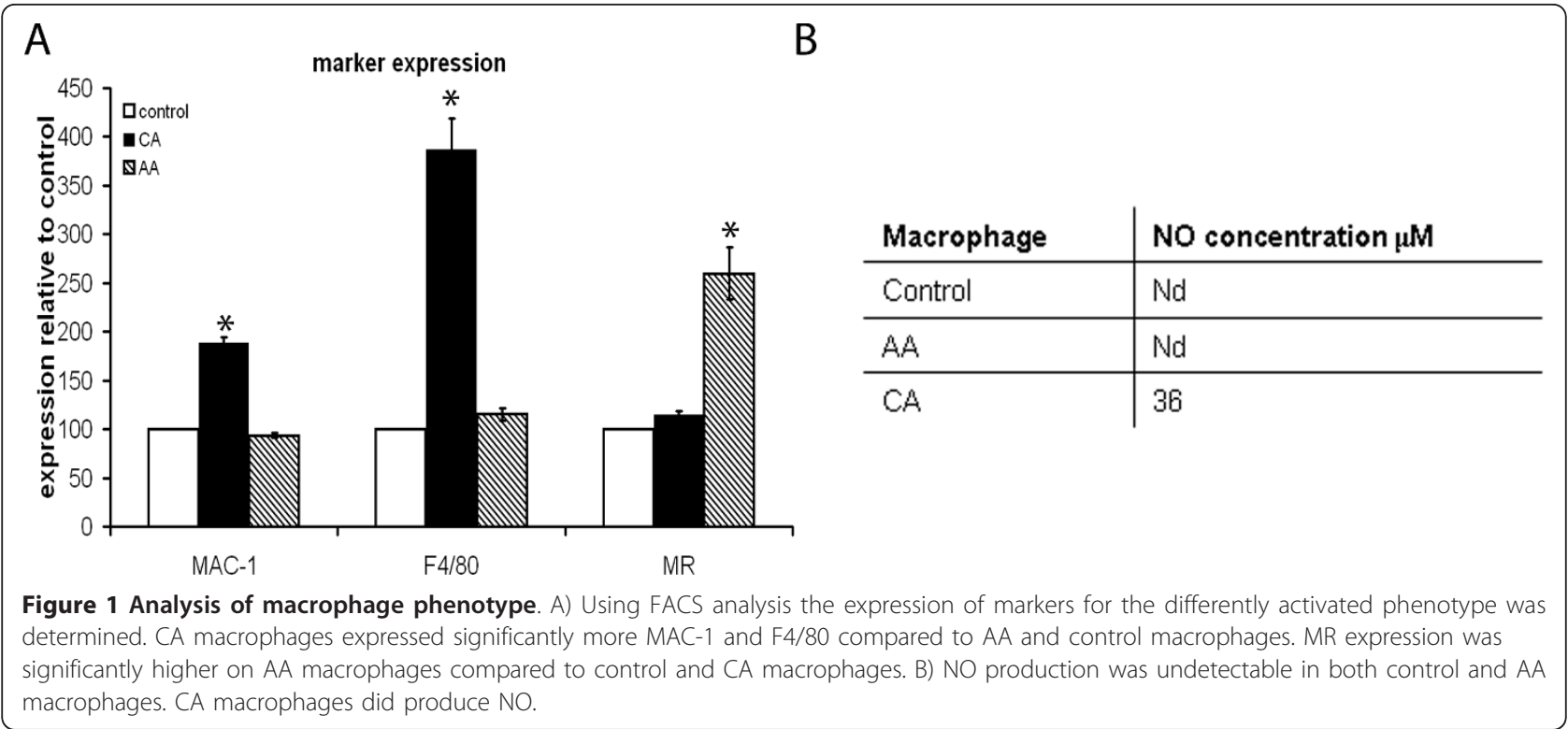

micro chemotaxis chamber was used. Macrophages migrated over a filter in response to conditioned medium harvested from neurons, astrocytes and several developmental stages of oligodendrocytes.

AA macrophages migrated in significantly higher numbers towards NCM compared to control neurobasal medium. CA macrophages were not attracted towards NCM (Figure 2A). In order to determine the molecular weight range of the factors that act chemotactic on AA macrophages, NCM was fractioned according to molecular weight. The NCM fraction containing proteins smaller than $10 \mathrm{kD}$ was responsible for this effect (Figure 2B).

In contrast, migration of CA macrophages was significantly enhanced towards ACM compared to control medium (Figure $2 \mathrm{C}$ ). The ACM fraction containing molecules smaller than $10 \mathrm{kD}$ was responsible for the attraction of CA macrophages (Figure 2D). Fractions containing proteins larger than $10 \mathrm{kD}$ did not significantly attract macrophages. AA macrophages did not migrate in higher numbers towards ACM compared to control medium.

The conditioned media of different stages of oligodendrocyte development, from precursors to mature oligodendrocytes, significantly attracted CA and AA macrophages compared to the control medium (Figure $2 \mathrm{E})$. Control macrophages migrated in significantly higher numbers towards the GC and MBP developmental stages compared to control medium. Specifically the GC developmental stage induced enhanced migration in all macrophage phenotypes. CA macrophages were attracted in significantly higher numbers compared to both control and AA macrophages.

\section{AA macrophages have a higher motility than CA macrophages}

Since we observed this striking effect on migration, we next determined the motility of CA and AA macrophages using a time-lapse video microscope. AA macrophages were significantly more motile compared to CA macrophages (Figure $3 \mathrm{~A}$ and $3 \mathrm{~B}$ ). CA macrophages appeared to move very little.

Since motility was lower in CA macrophages compared to AA macrophages, we wanted to determine whether the intrinsic migratory capacity of CA macrophages was also reduced. Migration towards the potent macrophage attractors MCP-1 and fMLP, a synthetic analogue of a bacterial signal peptide, was used to address this question. As can be seen in Figure 3C, AA and $C A$ macrophages migrated in comparable numbers towards $\mathrm{FMLP}$ and MCP-1, indicating that the intrinsic migratory capacity did not differ.

We next investigated whether the difference in motility was related to altered adherence. Therefore, the adhesion capacity of the differently activated macrophages to culture plastic and ECM molecules was measured. On culture plates CA macrophages displayed a trend towards increased adherence compared to control and AA macrophages (Figure 3D). After coating the culture plates with either collagen or fibronectin, the percentage of CA macrophages that adhered was enhanced twofold compared to both control and AA macrophages. We next investigated whether altered presence of adhesion receptors could explain the differences in adherence seen. We determined the expression of MAC-1, lymphocyte function associated antigen-1 (LFA-1), 


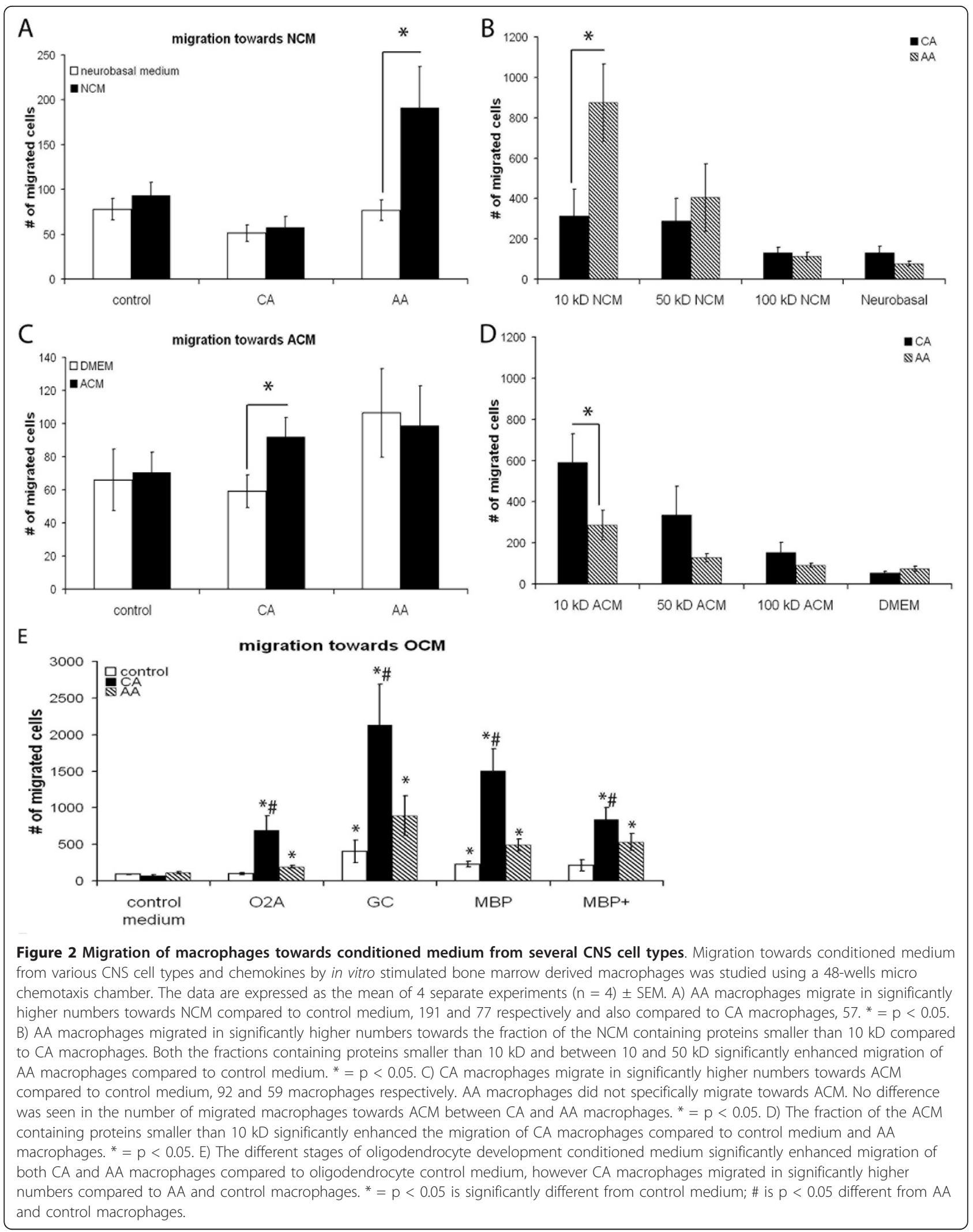



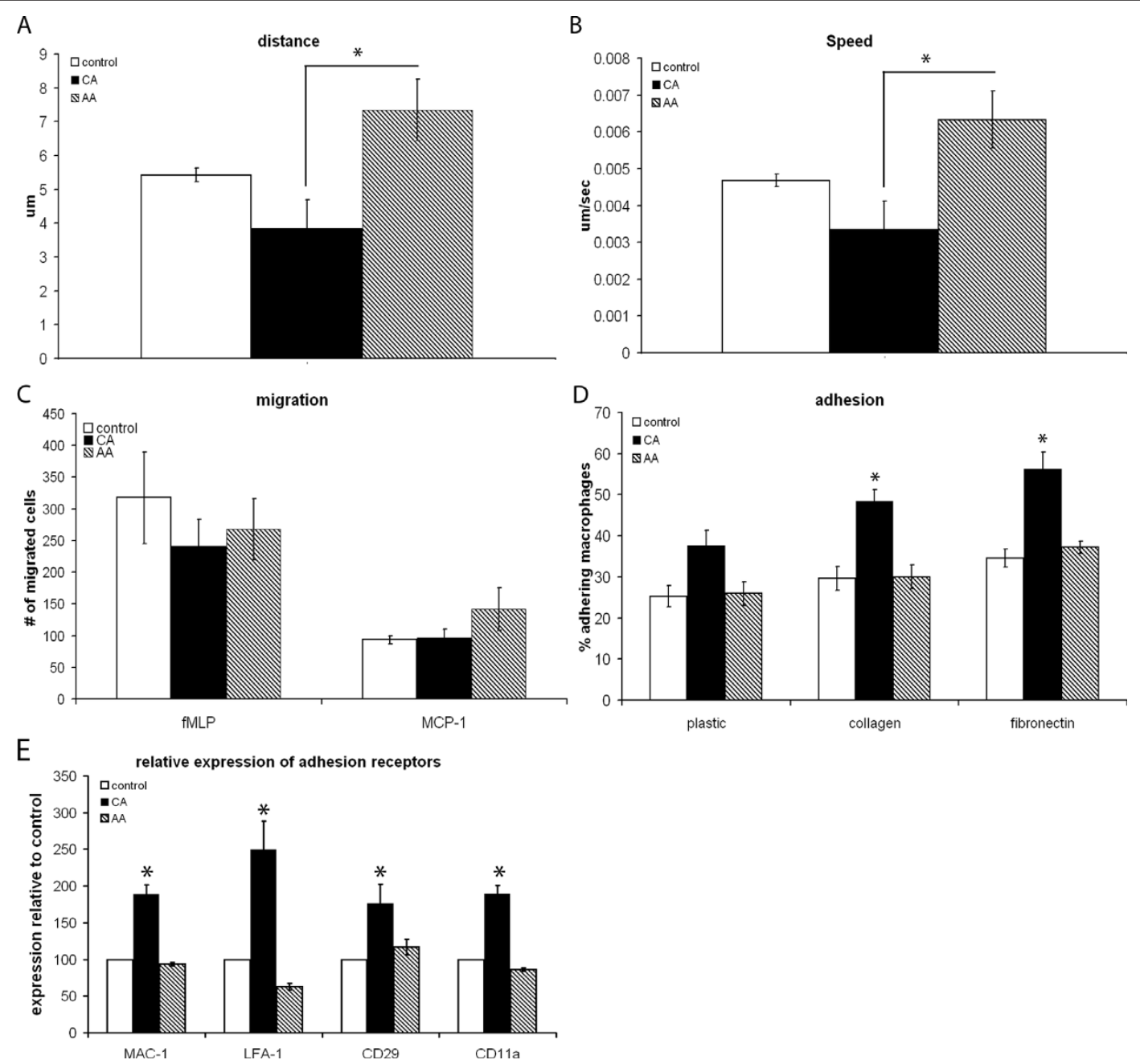

Figure 3 Motility and migratory capacity. Motility of in vitro stimulated bone marrow derived macrophages was determined by timelapse video microscopy. Speed and distance covered were determined using Tracklt software. The migration towards fMLP and MCP-1 was used as a measure for migratory capacity of in vitro stimulated bone marrow derived macrophages. Adhesion was determined by plating fluorescently labeled macrophages on (un)coated 96 wells plates, washing the plates after $2 \mathrm{~h}$ and measuring fluorescence. Data are the mean of 3 separate experiments $(n=3) \pm S E M .{ }^{*}=p<0.05$. A) During the recorded time the average distance AA macrophages covered was significantly enhanced twofold compared to CA macrophages. The distance was recorded in $\mu \mathrm{m}$. B) AA macrophages had a twofold higher speed compared to CA macrophages. Speed was recorded in $\mu \mathrm{m} / \mathrm{sec}$. C) No difference in migration was seen between the different macrophage phenotypes when comparing migration towards either FMLP or MCP-1. D) CA macrophages displayed a trend towards higher adherence on plastic compared to control and AA macrophages. On fibronectin and collagen adherence of CA macrophages was twofold higher compared to both control and AA macrophages. E) The expression of adhesion receptors was higher in CA macrophages compared to both AA and control macrophages.

CD29 and CD11a. CA macrophages expressed significantly more adhesion receptors compared to both control and AA macrophages (Figure 3E).

\section{Actin cytoskeleton of CA and AA macrophages}

Migration, motility and adhesion are functions that are all linked to the cytoskeletal organization of cells. Therefore we determined whether differences in the cytoskeleton could be observed between the macrophage phenotypes, by staining both phenotyopes for F-actin (Figure 4A-C). Remarkably, the morphology of CA macrophages appeared more rounded with an enhanced number of lamellipodia compared to control macrophages. In contrast, AA macrophages were elongated compared to control cells. In CA macrophages the staining pattern showed clustering of F-actin around the nucleus, whereas in AA macrophages the staining was more prominent at the border of the cell, indicating possible focal adhesions.

Next we determined the activity of small GTPases RhoA and Ras in activated macrophages, since they are involved in actin cytoskeleton modulation. The activity 

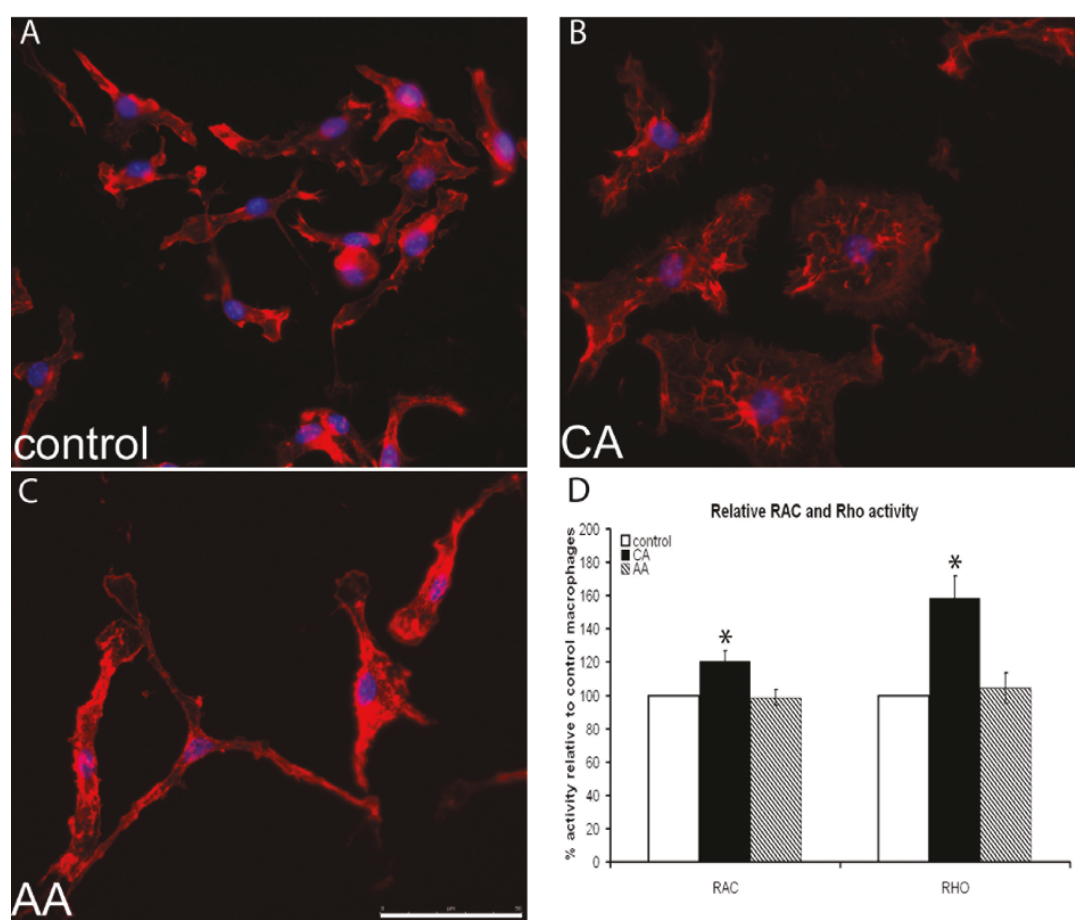

Figure 4 Cytoskeleton of differently activated macrophages. A-C) Visualization of actin cytoskeleton, red: actin (rhodamine phalloidin); blue: nuclear staining (Hoechst). Representative pictures are shown. CA macrophages appear flattened and rounded. AA macrophages are more stretched and elongated. Control macrophages appear to possess morphology in between the two extremes, with some cells being more elongated while others are more rounded. The scale bar is $50 \mu \mathrm{m}$ and a 40 times magnification was used. D) Rac and Rho activity. In CA macrophages small GTPases are more activated compared to both control and AA macrophages.

of both RhoA and Rac was significantly higher in CA macrophages compared to both control and AA macrophages (Figure 4D).

\section{Phagocytosis of myelin is higher in CA compared to AA macrophages}

Phagocytosis, a crucial macrophage function, is also linked to the activity of small GTPases and cytoskeletal reorganisation. We determined the myelin phagocytosis capacity of the differently activated macrophages.

CA macrophages had a significant twofold increase in the phagocytosis of myelin compared to both control and AA macrophages (Figure 5A). In order to ensure that phagocytosis had taken place, we showed internalization of myelin by confocal microscopy (Figure 5 B-D). Dil labeled myelin was visible within the green labeled membranes of macrophages, showing that phagocytosis of myelin had indeed taken place. Since Mac-1, also called CR3, has been shown to be involved in myelin phagocytosis [50] and we observed a significantly higher expression of Mac-1 by CA macrophages compared to both control and AA macrophages (Figure 1A and 3E) we determined whether phagocytosis of myelin could be blocked using anti-CR3 antibodies. Myelin phagocytosis could be blocked using anti-CR3 antibodies in CA macrophages and control macrophages. The block of myelin phagocytosis by anti-CR3 antibodies in AA macrophages was not significant (Figure 5B).

Next to CR3, ROS have also been implicated in the induction of phagocytosis. We therefore tested the effect a specific NADPH oxidase inhibitor, diphenylene iodonium (DPI), on myelin phagocytosis. Blocking ROS production, by DPI, reduced myelin phagocytosis by control and AA macrophages significantly (Figure 6). However, exposure to DPI did not have a significant effect on myelin phagocytosis by CA macrophages (Figure 6). Finally, flavonoids were used to block phagocytosis, since flavonoids are able to influence phagocytosis at multiple levels, such as ROS production and cytoskeleton mobilization. Using quercetin and luteolin, phagocytosis of myelin by CA, AA and control macrophages was blocked (Figure 6).

\section{Phagocytosis of neuronal fragments is higher in CA compared to AA macrophages}

During neuroinflammatory diseases not only myelin debris is present, but also neuronal debris. Phagocytosis of myelin is well investigated, which is not the case for neuronal debris. Indications that neuronal debris is phagocytosed, comes from MS research. Macrophages were suggested to transport neuronal debris outside the CNS, 
A

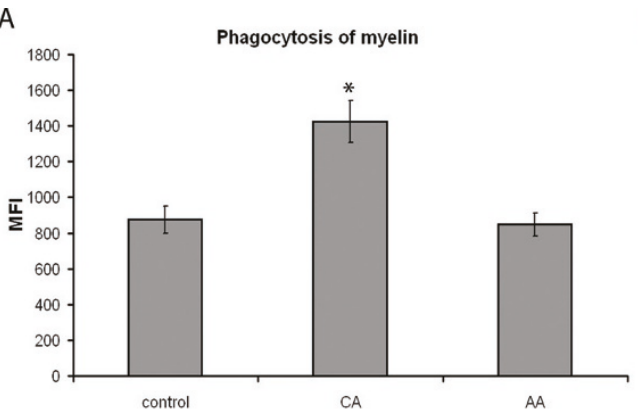

C

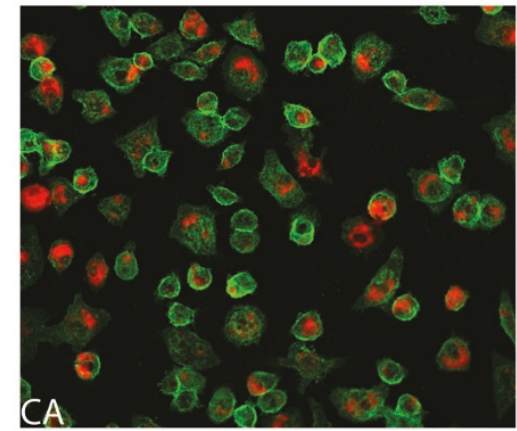

E

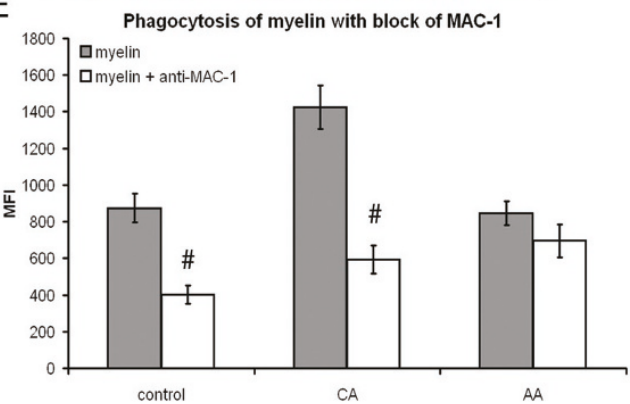

B

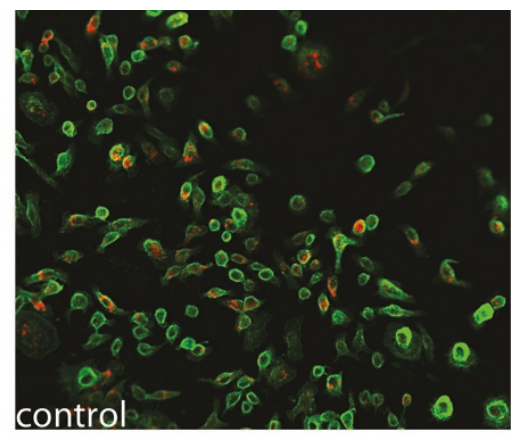

D

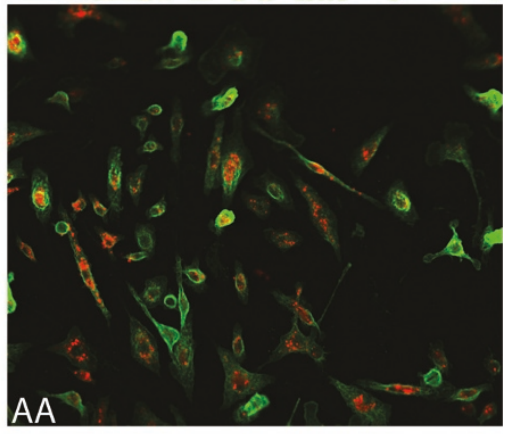

Figure 5 Phagocytosis of myelin by differently activated macrophages. Phagocytosis of fluorescently labeled myelin by activated bone marrow derived macrophages and expression of CR3 was determined using flow cytometry. MFI was measured and data are the mean of 4 separate experiments $(n=4) \pm$ SEM. ${ }^{*}=p<0.05$ and $\#=p<0.05$. A) CA macrophages phagocytosed significantly more myelin compared to control and AA macrophages. B-D) Visualization of myelin internalization by phagocytosis, red: Dil labeled myelin; green: Mac-1 on macrophage membrane. Representative images, taken on a confocal microscope, are shown. Red myelin is seen inside the green labeled membrane of the differently activated macrophages. All macrophage phenotypes appear to have taken up myelin. A 20 times magnification was used. E) Using anti-MAC-1 antibody, phagocytosis of myelin by CA and control macrophages could be blocked significantly, as indicated by \#. Anti-MAC-1 antibody did not affect myelin phagocytosis by AA macrophages.

since macrophages in cervical lymph nodes of MS patients contained neuronal antigens [51]. Therefore, we investigated the phagocytosis of neuronal fragments, obtained by roughly pipetting a monolayer of neurons as a model for neuronal debris, by differently activated macrophages.

Phagocytosis of neuronal fragments was significantly enhanced by CA macrophages compared to both control and AA macrophages (Figure 7A). The phagocytosis of neuronal fragments did not differ between control and AA macrophages. To ensure that phagocytosis had taken place, we investigated internalization of neuronal fragments by confocal microscopy (Figure 7 B-D). All macrophage phenotypes were able to phagocytose neuronal fragments, since red labeled neuronal fragments were present within green labeled membranes. Some binding of neuronal fragments outside the macrophage membrane was also visible. The phagocytosis of neuronal fragments could be significantly blocked using luteolin and quercetin in all macrophage subtypes (Figure 7E). MAC-1 did not reduce phagocytosis of neuronal fragments. DPI only reduced phagocytosis in control macrophages.

\section{Discussion}

In this study we focused on functional consequences of differential activation of macrophages for cytoskeletonassociated functions as migration, motility, adhesion and 


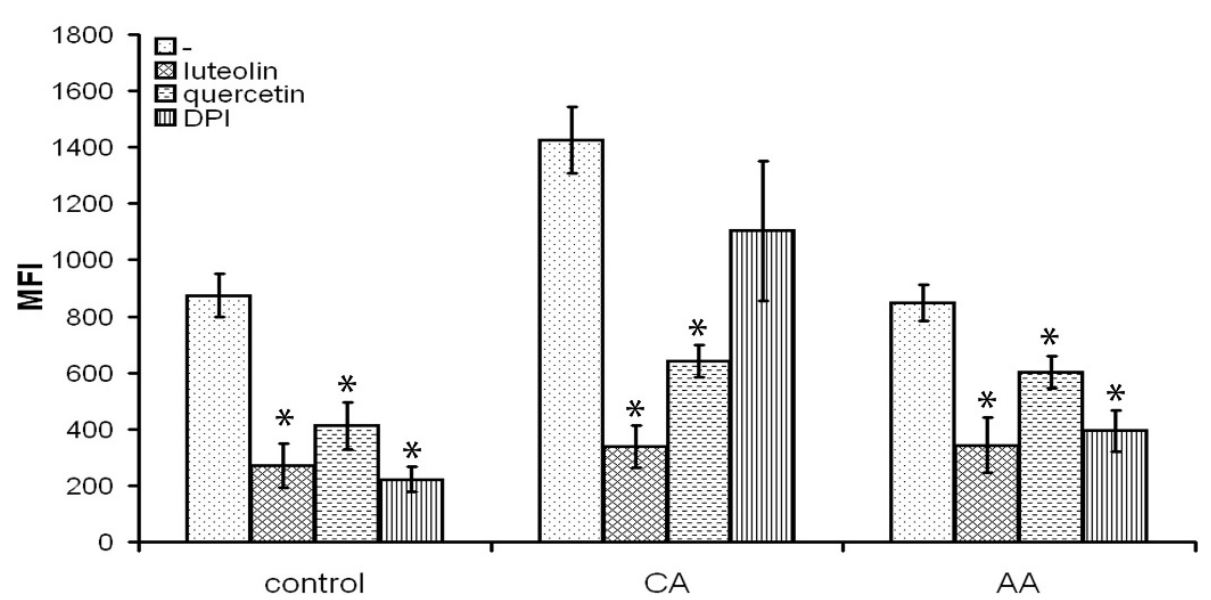

Figure 6 Influence of DPI and flavonoids on phagocytosis of myelin. To determine the influence of DPI, a NADPH oxidase blocker, and flavonoids on myelin phagocytosis, these substances were added together with fluorescently labeled myelin. Phagocytosis is determined as MFI of the macrophages. Data are the mean of 4 separate experiments $(n=4) \pm$ SEM. ${ }^{*}=p<0.05$. Both luteolin and quercetin significantly blocked phagocytosis of myelin by control, CA and AA macrophages. DPI only significantly reduced phagocytosis of myelin by control and AA

macrophages. DPI did not significantly decrease phagocytosis of myelin by CA macrophages.

phagocytosis in the CNS. These properties may have considerable impact on the local effects of macrophage subtypes during neuroinflammation. We observe that different CNS cell types have diverging effects on the migration of $\mathrm{CA}$ and AA macrophages. Furthermore we show that activation status affects motility, adhesion and phagocytosis of differently activated macrophages. Finally, the actin cytoskeleton appeared differently organized between CA and AA macrophages.

This study shows that AA and CA macrophages differ in the CNS cell types towards which they migrate. AA macrophages were significantly attracted towards NCM. Especially small proteins, $<10 \mathrm{kD}$, were responsible for the preferential attraction of CA versus AA macrophages, suggesting that chemokines could be responsible for this effect. Interestingly, human AA macrophages have increased expression levels of CXCR4, the receptor for CXCL12 which is expressed in cultured neurons [52] and in normal brain [53], while CA macrophages have higher expression levels of CCR7 [54]. Furthermore, the migration towards the $<10 \mathrm{kD}$ fraction of the medium induced a higher migration in AA macrophages compared to the whole medium, indicating that molecules present in the higher molecular weight fractions could be inhibiting migration. We observed that murine CA macrophages were significantly attracted towards ACM and oligodendrocyte conditioned medium. Using ACM, small proteins, $<10 \mathrm{kD}$, in the conditioned media attracted macrophages, suggesting that astrocytes release cytokines or chemokines. Again the lower molecular weight fraction of the medium induced a higher migration in CA macrophages compared to the complete medium, indicating the presence of inhibitory molecules in the whole medium. Finally, OCM, specifically of the GC developmental stage, attracted macrophages to a much higher extent compared to both NCM and ACM. The cells in the GC developmental stage are differentiating oligodendrocyte precursors. In vivo, macrophages are found both in areas of demyelination as well as in areas of remyelination. Furthermore, remyelination correlated with the number of oligodendrocytes and macrophages $[55,56]$. The fact that the OCM was derived from rat oligodendrocytes and the macrophages used for migration were murine could also play a role in the migration. Since in humans little research has been performed on differently activated macrophages within the CNS, no clinical data is available on the preferential association of differently activated macrophages with specific CNS cell types in human pathology.

Our results showed that the differences in migration were not due to intrinsic differences in migratory capacity, since fMLP and MCP-1 attracted both types of macrophages in comparable levels. Both fMLP and MCP-1 are potent chemoattractants [57,58]. Subtle differences in chemoattraction, due to differences in chemokine receptor expression, might be masked due to the high potency of these molecules.

One caveat in our study is that we used the supernatant of resting cultures to determine chemoattraction, which does not resemble the neuroinflammatory CNS. The cells in the inflamed CNS might secrete factors that were not present in our study, possibly changing the chemoattraction of the CA and AA macrophages. During EAE and MS, MCP-1 expression is upregulated in the CNS and in EAE a close association was observed between increased expression of MCP-1 and relapses 


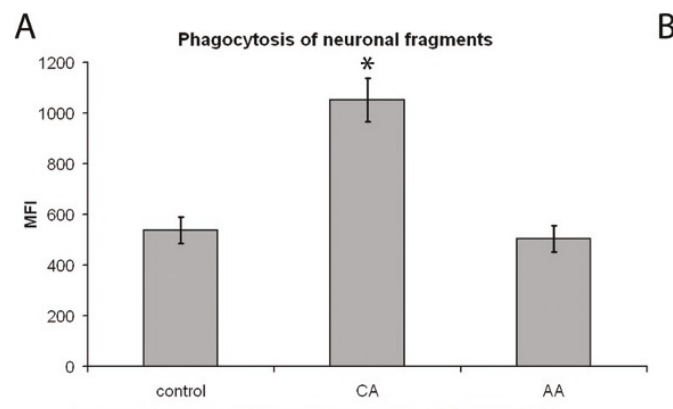

B

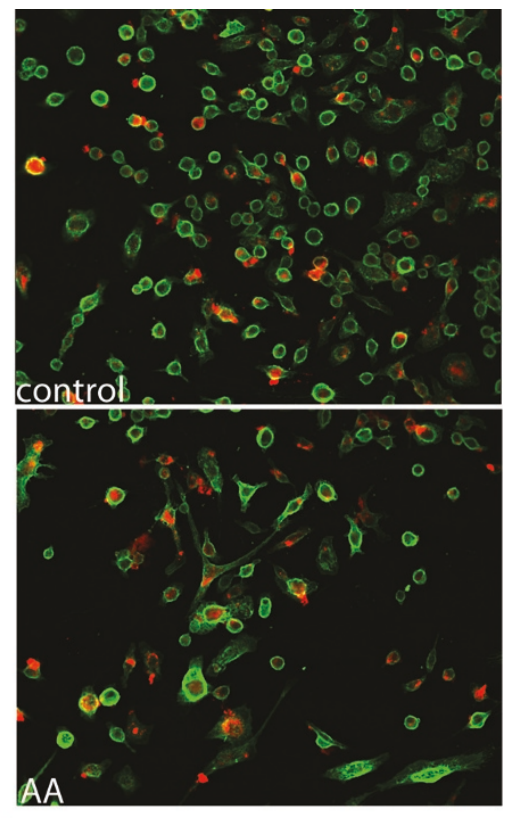

$\mathrm{E}$

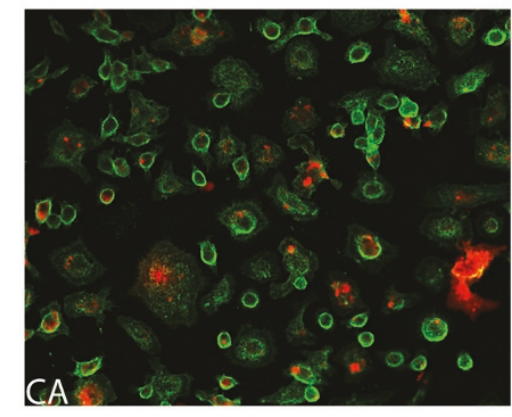

D

1200 Phagocytosis of neuronal fragments with block

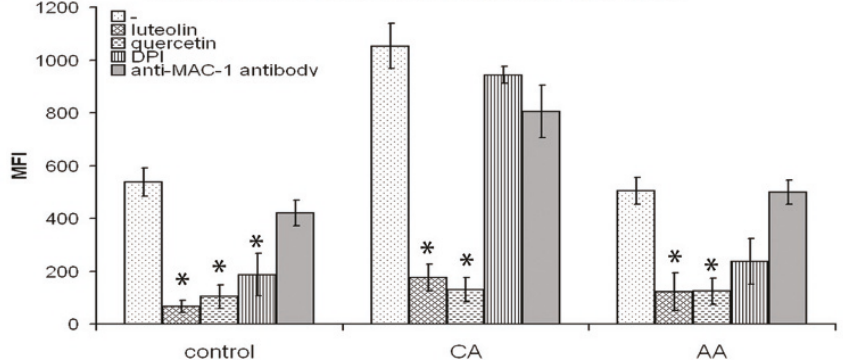

Figure 7 Phagocytosis of neuronal fragments by differently activated macrophages. Phagocytosis of fluorescently labeled neuronal fragments by activated bone marrow derived macrophages was determined using flow cytometry. Phagocytosis was blocked using anti-MAC-1 antibodies, luteolin, quercetin and DPI. Data are the mean of 4 separate experiments $(n=4) \pm$ SEM. ${ }^{*}=p<0.05$ and $\#=p<0.05$. A) CA macrophages phagocytosed significantly more neuronal fragments compared to control and AA macrophages. B-D) Visualization of neuronal fragment internalization by phagocytosis, red: Dil labeled neuronal fragments; green: Mac-1 on macrophage membrane. Representative images, taken on a confocal microscope (Leica TCS SP2), are shown. Red labelled neuronal fragments are clearly present within the green labelled membrane of the differently activated macrophages. Some binding is also present, with the neuronal fragments being present outside the membrane. A 20 times magnification was used. E) To determine whether phagocytosis could be blocked macrophages were exposed to luteolin, quercetin, MAC-1 (anti-CR3 antibody) or DPI (NADPH oxidase blocker). Phagocytosis of neuronal fragments by all types of macrophages was significantly reduced by exposure to luteolin and quercetin. Using MAC-1 antibody did not reduce neuronal fragment phagocytosis. DPI led to a significant reduction in control macrophages, however, CA and AA macrophages did not reduce phagocytosis significantly.

[59]. MCP-1 expression is mainly found in reactive astrocytes and macrophages [60,61]. Since MCP-1 attracted all subtypes of macrophages equally, this would indicate that in MS lesions all macrophage subtypes would be able to migrate equally towards these reactive astrocytes, since no differences were observed in migration towards MCP-1.

Although the intrinsic migratory capacity, determined using fMLP and MCP-1, did not differ between CA and AA macrophages, we observed significantly higher spontaneous motility in AA macrophages compared to CA macrophages. An important factor for motility is the adhesive capacity of macrophages. Adhesion can also be influenced by the cytoskeletal organization $[62,63]$. We showed that CA macrophages adhered to a higher extent to culture plates and ECM molecules compared to AA macrophages, confirming that adhesion might also have an impact on the increased motility and migration towards NCM of AA macrophages. Our findings were in line with a previous study, observing that glucocorticoid stimulated murine monocytes, which display some features of AA macrophages, adhered less and were more motile than control cells [64]. We observed enhanced expression adhesion receptors in CA macrophages. Lymphocyte function associated antigen-1 (LFA-1), MAC1 (CR3), CD29 and CD11a expression 
was higher in CA macrophages compared to AA macrophages. The increased expression of adhesion receptors in macrophages stimulated with IFN- $\gamma$ and LPS is in line with previous studies $[49,65]$ and with our current finding that CA macrophages adhere better when compared to AA macrophages.

We observed that AA macrophages were more elongated, whereas CA macrophages were rounded. This is in line with findings in human M1 and M2 macrophages. Human M2 macrophages, analogous to AA macrophages, appeared stretched with a spindle-like morphology, while human M1, analogous to CA macrophages, macrophages appeared rounder with a "friedegg" morphology [66]. The cytoskeletal organization differed between AA and CA macrophages, in that we found clusters of actin around the nucleus in CA macrophages, while the actin cytoskeleton of AA macrophages was more prominent at the border of the cell. Rho and Rac activity was higher in CA macrophages compared to both control and AA macrophages, possibly underlying the differences seen in cytoskeletal organization. These results indicate that during the transition to the AA phenotype macrophages become increasingly stretched and motile allowing migration into tissues, while transition to the CA phenotype leads to decreased motility and a round morphology, probably limiting migration.

Phagocytosis of myelin was higher in CA versus AA and control macrophages. We wanted to determine the mechanism behind this difference. CR3 is implicated in myelin phagocytosis [67]. We found a significantly higher expression of CR3, also called MAC-1, on CA macrophages compared to AA macrophages. This is in line with previous data showing upregulation of CR3 after IFN- $\gamma$ and LPS treatment of macrophages [49]. Myelin phagocytosis by CA macrophages could be blocked using anti-CR3 antibodies. A slight reduction in myelin phagocytosis was seen after exposure of AA macrophages to anti-CR3 antibodies. These results indicate that CR3 plays an important role in myelin phagocytosis by both control and CA macrophages, while in AA macrophages another mechanism of phagocytosis is dominant. Next to the phagocytosis of myelin, the CA macrophages also phagocytosed significantly more neuronal fragments compared to control and AA macrophages. CR3 is not involved in the phagocytosis of neuronal fragments, since addition of anti-CR3 antibodies did not reduce phagocytosis by any of the macrophage subtypes.

In rat macrophages myelin phagocytosis was ROS dependent [68]. Blocking ROS production by treatment with DPI blocked phagocytosis of myelin by AA and control macrophages, suggesting that ROS play an important role in myelin phagocytosis in these macrophages. The effect on CA macrophages was not significant, indicating that ROS do not play a significant role here. This could be due to the fact that myelin phagocytosis is CR-3 mediated in CA macrophages, while this does not seem to play a major role in either AA or control macrophages. Phagocytosis of neuronal fragments was reduced by DPI only in control macrophages, indicating an important role for ROS in control macrophage phagocytosis. Both CA and AA macrophages did not reduce phagocytosis of neuronal fragments after exposure to DPI, suggesting a specific mode of uptake similar to myelin uptake by CA macrophages via CR3.

The flavonoids quercetin and luteolin significantly reduced macrophage phagocytosis of both myelin and neuronal fragments by all types of macrophages. Previously, myelin phagocytosis was blocked after exposure to quercetin and luteolin in a macrophage cell line [69]. Quercetin and luteolin have anti-oxidant properties [69], suggesting that scavenging of ROS is an important factor in phagocytosis. Furthermore, they inhibit nuclear factor-kappa $\mathrm{B}$, thereby inhibiting pro-inflammatory cytokine secretion and NO production [70,71]. However, flavonoids do more than scavenge. Quercetin could also mediate its effects due to the inhibition of protein tyrosine kinases, which are involved in the signaling of the engulfment phase of CR3 mediated phagocytosis [72]. Luteolin has effects on the actin cytoskeleton $[73,74]$ through Rho and Rac [75] and could thereby affect phagocytosis.

In conclusion, the neuronal damage that occurs during neuroinflammatory diseases, such as MS, seems to be correlated to clinical disability $[27,76]$. CA macrophages have a lower ability to migrate, since they adhere strongly to the ECM and are generally less motile, limiting the amount of bystander damage due to ROS and NO secreted by the CA macrophages. Altogether this would contribute to a limited lesion expansion. Due to the fact that AA macrophages are more motile, adhere less to the ECM and are attracted by NCM, they could migrate towards neurons and locally release growth factors where they are most needed. AA macrophages are considered to be growth promoting and can secrete neurotrophic factors [30]. Therefore, skewing macrophages towards an AA phenotype could be a novel avenue for the development of new therapeutic strategies in neuroinflammatory diseases. Several studies have found therapeutic effects of AA macrophages. In vitro generated AA macrophages reduced renal and pancreatic injury in a model for murine diabetes [77]. In a model for colitis the injection of AA macrophages ameliorated the disease [78]. In spinal cord injury addition of multipotent adult progenitor cells reduced axonal dieback induced by macrophages [79]. In vitro these multipotent adult progenitor cells induced an AA phenotype in macrophages [79]. Treatment with anti-CCL22 [80] and 
2-arachidonoylglycerol [81], which were found to skew macrophages to an alternatively activated phenotype, increased the presence of AA macrophages in the lesions and ameliorated EAE disease course. Administration of AA macrophages reduced the development of relapses during the relapsing EAE model [82]. Adoptive transfer of AA macrophages induced using glatiramer acetate reversed clinical EAE [83]. Finally, in MS patients a deficiency in negative regulation of macrophage activation by Src homology region 2 domain-containing phosphatase-1 (SHP-1) was observed, leading to an enhanced response of these macrophages to both LPS and IL-4 $[84,85]$. This indicates that lesion environment determines the activation status of macrophages. Similar results have been found in a mouse model of Alzheimer's disease, since mRNA levels for markers of the CA and AA phenotype were observed to be upregulated in microglial cells, indicating that these microglia express functional characteristics of both $\mathrm{CA}$ and AA macrophages [86]. Together with the data from our current study, this supports the hypothesis that the "setting" of the innate immune system is crucial for disease outcome in inflammatory diseases of the CNS such as MS and SCI.

\section{List of abbreviations}

AA: alternatively activated; ACM: astrocyte conditioned medium; CA: classically activated; CNS: central nervous system; CR3: complement receptor 3; DMEM: Dulbecco modified Eagle's minimal essential medium; Dil: 1,1',dioctadecyl-3,3,3'3'-tetramethylindocarbocyanine perchlorate; DPI: diphenylene iodonium; EAE: experimental autoimmune encephalomyelitis; ECM: extracellular matrix; FCS: foetal calf serum; fMLP: formyl methionineleucylphenylalanine; GAP-43: growth associated protein 43; GFAP: glial fibrillary acidic protein; IFN-Y: interferon-gamma; IL: interleukin; iNOS: inducible nitric oxide synthetase; IR: immunoreactivity; LFA-1: lymphocyte function associated antigen-1; LPS: lipopolysaccharide; MBP: myelin basic protein; MCP-1: monocyte chemotactic peptide-1; MMP: matrix metalloproteinase; MR: mannose receptor; MS: multiple sclerosis; NADPH-oxidase: nicotinamide adenine dinucleotide phosphate-oxidase; NCM: neuronally conditioned medium; NF: neurofilament; NO: nitric oxide; OPC: oligodendrocyte precursor cell; PBS: phosphate buffered saline; ROS: reactive oxygen species; SCl: spinal cord injury

\section{Acknowledgements}

We would like to thank B. Beürger for providing mice and Martina Moeton and Ansam Barakat for technical assistance. This work was supported by the Dutch MS Research Foundation, grant number: 05-358c. Dutch Research Council (NWO (016.056.024)).

\section{Author details}

'Department of Molecular Cell Biology and Immunology, VU University Medical Center, Amsterdam, the Netherlands. ${ }^{2}$ University Medical Center Groningen University of Groningen, Dept. Cell Biology, Section Membrane Cell Biology, Groningen, the Netherlands. ${ }^{3}$ Department of Clinical chemistry, VU University Medical Center, Amsterdam, the Netherlands.

\section{Authors' contributions}

EV was involved in data acquisition, data analysis and statistical analysis. EV drafted the manuscript. DH performed the phagocytosis and FACS analysis assays. WB contributed the oligodendrocyte conditioned medium and critically reviewed the manuscript. HdV participated in the data analysis and conceptualization. CD and CT participated in study design, conceptualization, data analysis and helped to draft the manuscript. All authors have read and approved the final version of the manuscript.

\section{Competing interests}

The authors declare that they have no competing interests.

Received: 17 February 2011 Accepted: 26 May 2011

Published: 26 May 2011

\section{References}

1. Edwards JP, Zhang X, Frauwirth KA, Mosser DM: Biochemical and functional characterization of three activated macrophage populations. $J$ Leukoc Biol 2006, 80:1298-1307.

2. Mosser DM, Edwards JP: Exploring the full spectrum of macrophage activation. Nat Rev Immunol 2008, 8:958-969.

3. Stein M, Keshav S, Harris N, Gordon S: Interleukin 4 potently enhances murine macrophage mannose receptor activity: a marker of alternative immunologic macrophage activation. J Exp Med 1992, 176:287-292.

4. Nathan C, Shiloh MU: Reactive oxygen and nitrogen intermediates in the relationship between mammalian hosts and microbial pathogens. Proc Natl Acad Sci USA 2000, 97:8841-8848.

5. Kodelja V, Muller C, Tenorio S, Schebesch C, Orfanos CE, Goerdt S: Differences in angiogenic potential of classically vs alternatively activated macrophages. Immunobiology 1997, 197:478-493.

6. Mantovani A, Sica A, Allavena P, Garlanda C, Locati M: Tumor-associated macrophages and the related myeloid-derived suppressor cells as a paradigm of the diversity of macrophage activation. Hum Immunol 2009, 70:325-330.

7. Song E, Ouyang N, Horbelt M, Antus B, Wang M, Exton MS: Influence of alternatively and classically activated macrophages on fibrogenic activities of human fibroblasts. Cell Immunol 2000, 204:19-28.

8. Kreider T, Anthony RM, Urban JF, Gause WC: Alternatively activated macrophages in helminth infections. Curr Opin Immunol 2007, 19:448-453.

9. Albina JE, Mills CD, Henry WL, Caldwell MD: Temporal expression of different pathways of 1-arginine metabolism in healing wounds. J Immunol 1990, 144:3877-3880.

10. Gratchev A, Guillot P, Hakiy N, Politz O, Orfanos CE, Schledzewski K, Goerdt S: Alternatively activated macrophages differentially express fibronectin and its splice variants and the extracellular matrix protein betalG-H3. Scand I Immunol 2001, 53:386-392.

11. Hesse M, Modolell M, La Flamme AC, Schito M, Fuentes JM, Cheever AW, Pearce EJ, Wynn TA: Differential regulation of nitric oxide synthase-2 and arginase- 1 by type 1/type 2 cytokines in vivo: granulomatous pathology is shaped by the pattern of L-arginine metabolism. J Immunol 2001, 167:6533-6544.

12. Modolell M, Corraliza IM, Link F, Soler G, Eichmann K: Reciprocal regulation of the nitric oxide synthase/arginase balance in mouse bone marrowderived macrophages by TH1 and TH2 cytokines. Eur J Immunol 1995, 25:1101-1104

13. Lassmann $\mathrm{H}$, Bruck W, Lucchinetti $\mathrm{C}$, Rodriguez M: Remyelination in multiple sclerosis. Mult Scler 1997, 3:133-136.

14. van der Valk, De Groot CJ: Staging of multiple sclerosis (MS) lesions: pathology of the time frame of MS. Neuropathol Appl Neurobiol 2000, 26:2-10.

15. Cuzner ML, Gveric D, Strand C, Loughlin AJ, Paemen L, Opdenakker G, Newcombe J: The expression of tissue-type plasminogen activator, matrix metalloproteases and endogenous inhibitors in the central nervous system in multiple sclerosis: comparison of stages in lesion evolution. J Neuropathol Exp Neurol 1996, 55:1194-1204.

16. Sarchielli P, Greco L, Floridi A, Floridi A, Gallai V: Excitatory amino acids and multiple sclerosis: evidence from cerebrospinal fluid. Arch Neurol 2003, 60:1082-1088.

17. Bitsch A, Kuhlmann T, Da Costa C, Bunkowski S, Polak T, Bruck W: Tumour necrosis factor alpha mRNA expression in early multiple sclerosis lesions: correlation with demyelinating activity and oligodendrocyte pathology. Glia 2000, 29:366-375.

18. Smith KJ, Lassmann $\mathrm{H}$ : The role of nitric oxide in multiple sclerosis. Lancet Neurol 2002, 1:232-241

19. Conrad DJ, Kuhn H, Mulkins M, Highland E, Sigal E: Specific inflammatory cytokines regulate the expression of human monocyte 15-lipoxygenase. Proc Natl Acad Sci USA 1992, 89:217-221. 
20. Hendriks JJ, Teunissen CE, de Vries HE, Dijkstra CD: Macrophages and neurodegeneration. Brain Res Brain Res Rev 2005, 48:185-195.

21. Selmaj KW, Raine CS: Tumor necrosis factor mediates myelin and oligodendrocyte damage in vitro. Ann Neurol 1988, 23:339-346.

22. Newman TA, Woolley ST, Hughes PM, Sibson NR, Anthony DC, Perry VH: Tcell- and macrophage-mediated axon damage in the absence of a CNSspecific immune response: involvement of metalloproteinases. Brain 2001, 124:2203-2214.

23. Huitinga I, van Rooijen N, De Groot CJ, Uitdehaag BM, Dijkstra CD: Suppression of experimental allergic encephalomyelitis in Lewis rats after elimination of macrophages. J Exp Med 1990, 172:1025-1033.

24. Heppner FL, Greter M, Marino D, Falsig J, Raivich G, Hovelmeyer N, Waisman A, Rulicke T, Prinz M, Priller J, Becher B, Aguzzi A: Experimental autoimmune encephalomyelitis repressed by microglial paralysis. Nat Med 2005, 11:146-152.

25. Bitsch A, Schuchardt J, Bunkowski S, Kuhlmann T, Bruck W: Acute axonal injury in multiple sclerosis. Correlation with demyelination and inflammation. Brain 2000, 123:1174-1183.

26. Kuhlmann T, Lingfeld G, Bitsch A, Schuchardt J, Bruck W: Acute axonal damage in multiple sclerosis is most extensive in early disease stages and decreases over time. Brain 2002, 125:2202-2212.

27. Trapp BD, Peterson J, Ransohoff RM, Rudick R, Mork S, Bo L: Axonal transection in the lesions of multiple sclerosis. N Engl J Med 1998 338:278-285

28. Kotter MR, Li WW, Zhao C, Franklin RJ: Myelin impairs CNS remyelination by inhibiting oligodendrocyte precursor cell differentiation. J Neurosci 2006, 26:328-332.

29. Baer AS, Syed YA, Kang SU, Mitteregger D, Vig R, Ffrench-Constant C, Franklin RJ, Altmann F, Lubec G, Kotter MR: Myelin-mediated inhibition of oligodendrocyte precursor differentiation can be overcome by pharmacological modulation of Fyn-RhoA and protein kinase $\mathrm{C}$ signalling. Brain 2009, 132:465-481.

30. Kerschensteiner M, Gallmeier E, Behrens L, Leal W, Misgeld T, Klinkert WE, Kolbeck R, Hoppe E, Oropeza-Wekerle RL, Bartke I, Stadelmann C, Lassmann H, Wekerle $H$, Hohlfeld R: Activated human T cells, B cells, and monocytes produce brain-derived neurotrophic factor in vitro and in inflammatory brain lesions: a neuroprotective role of inflammation? J Exp Med 1999, 189:865-870.

31. Stadelmann C, Kerschensteiner M, Misgeld T, Bruck W, Hohlfeld R, Lassmann H: BDNF and gp145trkB in multiple sclerosis brain lesions: neuroprotective interactions between immune and neuronal cells? Brain 2002, 125:75-85.

32. Kotter MR, Zhao C, van Rooijen N, Franklin RJ: Macrophage-depletion induced impairment of experimental CNS remyelination is associated with a reduced oligodendrocyte progenitor cell response and altered growth factor expression. Neurobiol Dis 2005, 18:166-175.

33. Popovich PG, Guan Z, Wei P, Huitinga I, van Rooijen N, Stokes BT: Depletion of hematogenous macrophages promotes partial hindlimb recovery and neuroanatomical repair after experimental spinal cord injury. Exp Neurol 1999, 158:351-365.

34. Stirling DP, Khodarahmi K, Liu J, McPhail LT, McBride CB, Steeves JD Ramer MS, Tetzlaff W: Minocycline treatment reduces delayed oligodendrocyte death, attenuates axonal dieback, and improves functional outcome after spinal cord injury. J Neurosci 2004, 24:2182-2190.

35. lannotti CA, Clark M, Horn KP, van RN, Silver J, Steinmetz MP: A combination immunomodulatory treatment promotes neuroprotection and locomotor recovery after contusion SCI. Exp Neurol 2010.

36. Rapalino O, Lazarov-Spiegler O, Agranov E, Velan GJ, Yoles E, Fraidakis M, Solomon A, Gepstein R, Katz A, Belkin M, Hadani M, Schwartz M: Implantation of stimulated homologous macrophages results in partial recovery of paraplegic rats. Nat Med 1998, 4:814-821.

37. Barrette B, Hebert MA, Filali M, Lafortune K, Vallieres N, Gowing G, Julien JP, Lacroix S: Requirement of myeloid cells for axon regeneration. J Neurosci 2008, 28:9363-9376.

38. Prewitt CM, Niesman IR, Kane CJ, Houle JD: Activated macrophage/ microglial cells can promote the regeneration of sensory axons into the injured spinal cord. Exp Neurol 1997, 148:433-443.

39. Kigerl KA, Gensel JC, Ankeny DP, Alexander JK, Donnelly DJ, Popovich PG: Identification of two distinct macrophage subsets with divergent effects causing either neurotoxicity or regeneration in the injured mouse spinal cord. J Neurosci 2009, 29:13435-13444.
40. Butovsky O, Ziv Y, Schwartz A, Landa G, Talpalar AE, Pluchino S, Martino G, Schwartz M: Microglia activated by IL-4 or IFN-gamma differentially induce neurogenesis and oligodendrogenesis from adult stem/ progenitor cells. Mol Cell Neurosci 2006, 31:149-160.

41. Ponomarev ED, Maresz K, Tan Y, Dittel BN: CNS-derived interleukin-4 is essential for the regulation of autoimmune inflammation and induces a state of alternative activation in microglial cells. J Neurosci 2007, 27:10714-10721

42. Johnson CR, Kitz D, Little JR: A method for the derivation and continuous propagation of cloned murine bone marrow macrophages. J Immunol Methods 1983, 65:319-332.

43. Varin A, Mukhopadhyay S, Herbein G, Gordon S: Alternative activation of macrophages by IL- 4 impairs phagocytosis of pathogens but potentiates microbial-induced signalling and cytokine secretion. Blood 2010, 115:353-362

44. Maier O, van der Heide T, van Dam AM, Baron W, de Vries H, Hoekstra D: Alteration of the extracellular matrix interferes with raft association of neurofascin in oligodendrocytes. Potential significance for multiple sclerosis. Mol Cell Neurosci 2005, 28:390-401.

45. Buttery PC, Ffrench-Constant C: Laminin-2/integrin interactions enhance myelin membrane formation by oligodendrocytes. Mol Cell Neurosci 1999, 14:199-212.

46. Bos HJ, Boorsma DM, Tuk CW, de Veld JC, Van der Muysenberg AJ, Helmerhorst TJ, Struijk DG, Van Bronswijk H, Beelen RH: Chemotaxis of the peritoneal cells and the detection of a chemo-attractant in the effluent from peritoneal dialysis patients. Eur J Clin Invest 1990, 20:555-562.

47. van der Goes A, Wouters D, van der Pol SM, Huizinga R, Ronken E, Adamson P, Greenwood J, Dijkstra CD, de Vries HE: Reactive oxygen species enhance the migration of monocytes across the blood-brain barrier in vitro. FASEB J 2001, 15:1852-1854.

48. Heeroma JH, Roelandse M, Wierda K, van Aerde Kl, Toonen RF, Hensbroek RA, Brussaard A, Matus A, Verhage M: Trophic support delays but does not prevent cell-intrinsic degeneration of neurons deficient for munc18-1. Eur J Neurosci 2004, 20:623-634.

49. Pourshafie MR, Sonnenfeld G: Treatment of an infected murine macrophage cell line (J774A.1) with interferon-gamma but not tumor necrosis factor-alpha or live Mycobacterium intracellulare alone modulates the expression of adhesion molecules. J Interferon Cytokine Res 1997, 17:69-75.

50. van der Laan LJ, Ruuls SR, Weber KS, Lodder IJ, Dopp EA, Dijkstra CD: Macrophage phagocytosis of myelin in vitro determined by flow cytometry: phagocytosis is mediated by CR3 and induces production of tumor necrosis factor-alpha and nitric oxide. J Neuroimmunol 1996, 70:145-152.

51. van Zwam $M$, Huizinga $R$, Melief MJ, Wierenga-Wolf AF, van Meurs $M$, Voerman JS, Biber KP, Boddeke HW, Hopken UE, Meisel C, Meisel A, Bechmann I, Hintzen RQ, 't Hart BA, Amor S, Laman JD, Boven LA: Brain antigens in functionally distinct antigen-presenting cell populations in cervical lymph nodes in MS and EAE. J Mol Med 2009, 87:273-286.

52. de Haas AH, van Weering HR, de Jong EK, Boddeke HW, Biber KP: Neuronal chemokines: versatile messengers in central nervous system cell interaction. Mol Neurobiol 2007, 36:137-151.

53. Gleichmann M, Gillen C, Czardybon M, Bosse F, Greiner-Petter R, Auer J, Muller HW: Cloning and characterization of SDF-1gamma, a novel SDF-1 chemokine transcript with developmentally regulated expression in the nervous system. Eur J Neurosci 2000, 12:1857-1866.

54. Martinez FO, Gordon S, Locati M, Mantovani A: Transcriptional profiling of the human monocyte-to-macrophage differentiation and polarization: new molecules and patterns of gene expression. J Immunol 2006, 177:7303-7311.

55. Wolswijk G: Oligodendrocyte precursor cells in the demyelinated multiple sclerosis spinal cord. Brain 2002, 125:338-349.

56. Kotter MR, Setzu A, Sim FJ, van Rooijen N, Franklin RJ: Macrophage depletion impairs oligodendrocyte remyelination following lysolecithininduced demyelination. Glia 2001, 35:204-212

57. Schiffmann E: Leukocyte chemotaxis. Annu Rev Physiol 1982, 44:553-568

58. Deshmane SL, Kremlev S, Amini S, Sawaya BE: Monocyte chemoattractant protein-1 (MCP-1): an overview. J Interferon Cytokine Res 2009, 29:313-326.

59. Jee Y, Yoon WK, Okura Y, Tanuma N, Matsumoto Y: Upregulation of monocyte chemotactic protein- 1 and CC chemokine receptor 2 in the 
central nervous system is closely associated with relapse of autoimmune encephalomyelitis in Lewis rats. J Neuroimmunol 2002, 128:49-57.

60. Berman JW, Guida MP, Warren J, Amat J, Brosnan CF: Localization of monocyte chemoattractant peptide-1 expression in the central nervous system in experimental autoimmune encephalomyelitis and trauma in the rat. I Immunol 1996, 156:3017-3023.

61. Simpson JE, Newcombe J, Cuzner ML, Woodroofe MN: Expression of monocyte chemoattractant protein- 1 and other beta-chemokines by resident glia and inflammatory cells in multiple sclerosis lesions. J Neuroimmunol 1998, 84:238-249.

62. Ke H, Parron VI, Reece J, Zhang JY, Akiyama SK, French JE: BCL2 inhibits cell adhesion, spreading, and motility by enhancing actin polymerization. Cell Res 2010, 20:458-469.

63. Lochhead PA, Wickman G, Mezna M, Olson MF: Activating ROCK1 somatic mutations in human cancer. Oncogene 2010, 29:2591-2598.

64. Varga G, Ehrchen J, Tsianakas A, Tenbrock K, Rattenholl A, Seeliger S, Mack M, Roth J, Sunderkoetter C: Glucocorticoids induce an activated, anti-inflammatory monocyte subset in mice that resembles myeloidderived suppressor cells. J Leukoc Biol 2008, 84:644-650.

65. Goebeler M, Roth J, Kunz M, Sorg C: Expression of intercellular adhesion molecule-1 by murine macrophages is up-regulated during differentiation and inflammatory activation. Immunobiology 1993, 188:159-171.

66. Verreck FA, de Boer T, Langenberg DM, van der Zanden L, Ottenhoff TH: Phenotypic and functional profiling of human proinflammatory type-1 and anti-inflammatory type-2 macrophages in response to microbial antigens and IFN-gamma- and CD40L-mediated costimulation. J Leukoc Biol 2006, 79:285-293.

67. van der Laan L, Ruuls SR, Weber KS, Lodder IJ, Dopp EA, Dijkstra CD: Macrophage phagocytosis of myelin in vitro determined by flow cytometry: phagocytosis is mediated by CR3 and induces production of tumor necrosis factor-alpha and nitric oxide. J Neuroimmunol 1996, 70:145-152.

68. van der Goes A, Brouwer J, Hoekstra K, Roos D, van den Berg TK, Dijkstra CD: Reactive oxygen species are required for the phagocytosis of myelin by macrophages. J Neuroimmunol 1998, 92:67-75.

69. Hendriks JJ, de Vries HE, van der Pol SM, Van Den Berg TK, van Tol EA, Dijkstra CD: Flavonoids inhibit myelin phagocytosis by macrophages; a structure-activity relationship study. Biochem Pharmacol 2003, 65:877-885.

70. Kim HK, Cheon BS, Kim YH, Kim SY, Kim HP: Effects of naturally occurring flavonoids on nitric oxide production in the macrophage cell line RAW 264.7 and their structure-activity relationships. Biochem Pharmacol 1999, 58:759-765.

71. Wadsworth TL, Koop DR: Effects of the wine polyphenolics quercetin and resveratrol on pro-inflammatory cytokine expression in RAW 264.7 macrophages. Biochem Pharmacol 1999, 57:941-949.

72. Hespanhol MR, Mantovani B: Phagocytosis by macrophages mediated by receptors for denatured proteins - dependence on tyrosine protein kinases. Braz J Med Biol Res 2002, 35:383-389.

73. Lee WJ, Wu LF, Chen WK, Wang CJ, Tseng TH: Inhibitory effect of luteolin on hepatocyte growth factor/scatter factor-induced HepG2 cell invasion involving both MAPK/ERKs and PI3K-Akt pathways. Chem Biol Interact 2006, 160:123-133.

74. Lee JW, Ahn JY, Hasegawa S, Cha BY, Yonezawa T, Nagai K, Seo HJ, Jeon WB, Woo JT: Inhibitory effect of luteolin on osteoclast differentiation and function. Cytotechnology 2009, 61:125-134.

75. Hendriks JJ, Alblas J, van der Pol SM, van Tol EA, Dijkstra CD, de Vries HE: Flavonoids influence monocytic GTPase activity and are protective in experimental allergic encephalitis. J Exp Med 2004, 200:1667-1672.

76. Bjartmar C, Kidd G, Mork S, Rudick R, Trapp BD: Neurological disability correlates with spinal cord axonal loss and reduced $\mathrm{N}$-acetyl aspartate in chronic multiple sclerosis patients. Ann Neurol 2000, 48:893-901.

77. Zheng D, Wang Y, Cao Q, Lee WW, Zheng G, Sun Y, Tan TK, Wang Y, Alexander SI, Harris DC: Transfused Macrophages Ameliorate Pancreatic and Renal Injury in Murine Diabetes Mellitus. Nephron Exp Nephrol 2011, 118:e87-e99.

78. Hunter MM, Wang A, Parhar KS, Johnston MJ, van RN, Beck PL, McKay DM: In vitro-derived alternatively activated macrophages reduce colonic inflammation in mice. Gastroenterology 2010, 138:1395-1405.

79. Busch SA, Hamilton JA, Horn KP, Cuascut FX, Cutrone R, Lehman N, Deans RJ, Ting AE, Mays RW, Silver J: Multipotent adult progenitor cells prevent macrophage-mediated axonal dieback and promote regrowth after spinal cord injury. J Neurosci 2011, 31:944-953.

80. Dogan RN, Long N, Forde E, Dennis K, Kohm AP, Miller SD, Karpus WJ: CCL22 regulates experimental autoimmune encephalomyelitis by controlling inflammatory macrophage accumulation and effector function. J Leukoc Biol 2011, 89:93-104.

81. Lourbopoulos A, Grigoriadis N, Lagoudaki R, Touloumi O, Polyzoidou E, Mavromatis I, Tascos N, Breuer A, Ovadia H, Karussis D, Shohami E, Mechoulam R, Simeonidou C: Administration of 2-arachidonoylglycerol ameliorates both acute and chronic Experimental Autoimmune Encephalomyelitis. Brain Res 2011, 1390:126-41.

82. Mikita J, Dubourdieu-Cassagno N, Deloire MS, Vekris A, Biran M, Raffard G, Brochet B, Canron MH, Franconi JM, Boiziau C, Petry KG: Altered M1/M2 activation patterns of monocytes in severe relapsing experimental rat model of Multiple Sclerosis. Amelioration of clinical status by M2 activated monocyte administration. Mult Scler 2011, 17(1):2-15.

83. Weber MS, Prod'homme T, Youssef S, Dunn SE, Rundle CD, Lee L, Patarroyo JC, Stuve O, Sobel RA, Steinman L, Zamvil SS: Type II monocytes modulate $T$ cell-mediated central nervous system autoimmune disease. Nat Med 2007, 13:935-943.

84. Christophi GP, Panos M, Hudson CA, Christophi RL, Gruber RC, Mersich AT Blystone SD, Jubelt B, Massa PT: Macrophages of multiple sclerosis patients display deficient SHP-1 expression and enhanced inflammatory phenotype. Lab Invest 2009, 89:742-759.

85. Christophi GP, Hudson CA, Gruber RC, Christophi CP, Mihai C, Mejico LJ, Jubelt B, Massa PT: SHP-1 deficiency and increased inflammatory gene expression in PBMCs of multiple sclerosis patients. Lab Invest 2008, 88:243-255.

86. Colton CA, Mott RT, Sharpe $H, X u$ Q, Van Nostrand WE, Vitek MP: Expression profiles for macrophage alternative activation genes in $A D$ and in mouse models of AD. J Neuroinflammation 2006, 3:27.

doi:10.1186/1742-2094-8-58

Cite this article as: Vereyken et al: Classically and alternatively activated bone marrow derived macrophages differ in cytoskeletal functions and migration towards specific CNS cell types. Journal of Neuroinflammation $20118: 58$

\section{Submit your next manuscript to BioMed Central and take full advantage of:}

- Convenient online submission

- Thorough peer review

- No space constraints or color figure charges

- Immediate publication on acceptance

- Inclusion in PubMed, CAS, Scopus and Google Scholar

- Research which is freely available for redistribution

Submit your manuscript at www.biomedcentral.com/submit
Ciomed Central 